\title{
About Optimal Performance and Approximation of Open-Channel Hydraulic Systems
}

\author{
Xavier Litrico \\ Cemagref, UR IRMO \\ B.P. 5095, 34033 Montpellier Cedex 1, France \\ e-mail: xavier.litrico@cemagref.fr
}

\author{
Vincent Fromion \\ LASB, INRA Montpellier \\ 2 place Viala, 34060 Montpellier, France \\ e-mail: fromion@ensam.inra.fr
}

\begin{abstract}
The article investigates the optimal performance of feedback controlled open-channel systems represented by a linear advection-diffusion equation. We show that the performance given by the maximal achievable bandwidth over which the sensitivity function stays below one for such a system is structurally limited. This result is obtained using different tools. Firstly, an approximate value is computed with stability considerations. A close result is also obtained putting the problem in the $H_{\infty}$ framework. We finally compare the previous results with a qualitative approach using Bode's ideal loop transfer function.
\end{abstract}

\section{Introduction}

Faced to the increasing demand in water savings, hydraulic engineers use automatic control techniques in order to obtain a better performance in real-time operation of open-channel systems. The open-channel system considered are made of a network of river reaches controlled by a finite number of dams situated upstream. The overall system forms a multiple-input multiple-output system, which is the interconnection of several single-input single-output sub-systems. It can be proved that the performance of the MIMO system which possesses a specific structure can be linked to the one of the SISO sub-systems. The paper will therefore focus on the analysis of a single reach represented by a SISO transfer function. The discharge is supposed to be measured at the downstream end of the river reach and there is a finite number of intermediate pumping stations distributed along the reach which provide water to consumers (typically farmers who irrigate fields). The objective of the controller is to keep the measured downstream discharge close to a target despite unmeasured users' withdrawals.

The dynamics of these open-channel hydraulic systems are represented by a partial derivative equation, or in the frequency domain by an irrational transfer function.
The aim of this paper is to show that the achievable performance of such open-channel hydraulic systems is structurally limited. As the system is subject to unmeasured perturbations acting at the output, we seek the maximum frequency range where attenuation can be obtained. To this purpose, we examine classical limitations as Bode integral constraints [9] and show that they do not constraint in direct way the achievable sensitivity. In fact, we show that the stability constraint limits the bandwidth like in cases of delayed systems [11].

Another way to study the considered problem is to put it into the $H_{\infty}$ framework. Recent work allow to solve the sensitivity problem for irrational systems (see e.g. $[13,15,5]$ and references therein). Unfortunately, the required conditions in [15] are not satisfied in our case. We then consider the approach proposed in [14] where the solutions are obtained using finite dimensional approximations of the irrational system and computation of classical $H_{\infty}$ solution for rational systems.

The last part of the paper proposes a more realistic design with high frequencies constraints on the input. This "mixed sensitivity" criteria allows to take into account actuator limitations and delay margin requirements. We end the paper by a remark on the interest of the heuristics associated to Bode's ideal loop transfer.

\section{Problem statement}

\subsection{Considered problem}

The system considered in this paper is a controlled river where the action variable is the upstream discharge and the measured variable is the downstream discharge (see figure 1). The river is used to deliver water from the upstream dam to various consumers pumping water along the reach (farmers irrigating their fields, industries, etc.). The objective of the controller is to keep the measured downstream discharge close to a target despite unmeasured users' withdrawals.

In other terms, the control objective is to use the upstream discharge $u$ in order to keep the downstream 


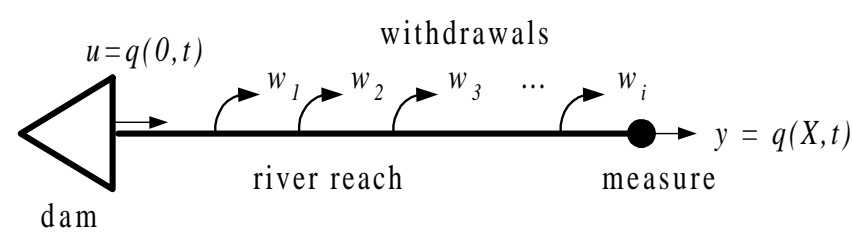

Figure 1: Dam-river system considered

discharge $y$ as constant as possible, which means that the control should attenuate the unmeasured perturbations $w$. This is a problem of regulation or desensitivity. This objective should be attained with minimum gain and phase margins.

As the perturbations $w_{i}$ are all unmeasured, they can be aggregated into one point without loss of generality. The system is then represented with all the withdrawals gathered at the downstream end by the equation:

$$
y=G(s) u+H(s) w
$$

with $H(s)$ the transfer function for the withdrawals $w$, $y=q(X, s), u=q(0, s)$ and $G(s)$ corresponding to the Hayami transfer function.

\subsection{Hayami model}

The Hayami equation is a linear partial derivative equation representing the discharge transfer in a river reach around an equilibrium point:

$$
\frac{\partial q}{\partial t}+C \frac{\partial q}{\partial x}-D \frac{\partial^{2} q}{\partial x^{2}}=0
$$

where

- $q$ is the discharge $\left[\mathrm{m}^{3} / \mathrm{s}\right]$,

- $C$ the celerity coefficient $[\mathrm{m} / \mathrm{s}]$ and

- $D$ the diffusion coefficient $\left[\mathrm{m}^{2} / \mathrm{s}\right]$.

This equation is obtained by linearizing the so-called diffusive wave equation around a reference discharge $Q_{r}$ [4]. Parameters $C$ and $D$ can be expressed as functions of $Q_{r}$ and other physical parameters of the river reach [12]. For a uniform large rectangular river reach, one gets:

$$
C=\frac{5 I^{0.3} Q_{r}^{0.4}}{3 L^{0.4} n^{0.6}} \text { and } D=\frac{Q_{r}}{2 L I}
$$

with $I$ the slope of the reach, $L$ the width, $n$ the Manning coefficient.

The relation between upstream and downstream discharge can also be expressed as a transfer function using Laplace transform. Thus, fixing a downstream limit condition of the type $\lim _{x \rightarrow \infty} \frac{\partial q}{\partial x}=0$, one gets the Hayami transfer function:

$$
G(s)=e^{\left(\frac{C-\sqrt{C^{2}+4 D s}}{2 D}\right) X}
$$

with $X$ the length of the reach $[\mathrm{m}]$ and $s$ the Laplace variable.

The river system considered in the applications throughout the paper is a $10 \mathrm{~km}$ long river with a uniform large rectangular geometry (width $L=8 \mathrm{~m}$, slope $I=0.0005$, Manning coefficient $n=0.05)$ and $Q_{r}=2$ $\mathrm{m}^{3} / \mathrm{s}$.

\section{Remarks:}

1. It can be shown that $G(s)$ is stable and belongs to the class of transfer functions defined by Callier and Desoer [3].

2. A first order with delay is a good approximation of the Hayami transfer function for low frequencies. This approximation is usually considered in hydrology [6]. As shown in figure 2, this approximation is rather crude concerning the group delay ${ }^{1}$, which decreases with frequency.

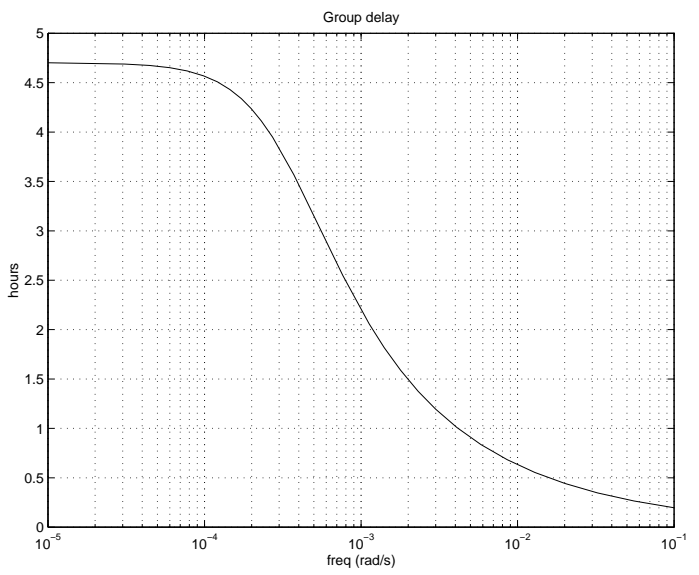

Figure 2: Group delay of $G(s)$

\section{Obtaining maximal performance bounds}

In this section, we address the following question: "What is the best achievable performance in perturbations attenuation?". In our context, we try to attenuate perturbations acting on the output of the system (see figure 3). This requirement can be formalized by direct constraints on the output sensitivity function. The performance considered is the maximum frequency $\omega_{s}$ such that the output sensitivity function $S=(1+G K)^{-1}$ stays below one

$$
\omega_{s}=\max \left\{\omega_{1}:|S(j \omega)|<1, \forall \omega<\omega_{1}\right\}
$$

\footnotetext{
${ }^{1}$ The group delay of $G(j \omega)$ is defined by $-\frac{\partial \angle G(j \omega)}{\partial \omega}[10]$.
} 
In this section, we first examine the performance limitations induced by the group delay by looking at the Bode sensitivity and stability. We secondly propose an $H_{\infty}$ criteria which incorporates more realistic design constraints and a way to obtain a rational controller which is an approximation of the optimal one.

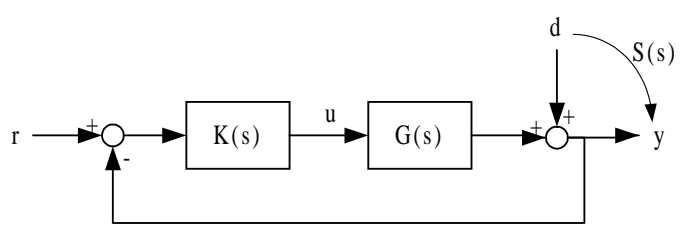

Figure 3: Feedback system with output perturbation

\subsection{Approximate value of $\omega_{s}$}

For systems described by rational transfer functions, the structural limitation of performance is now clearly understood (see e.g. $[2,8,1]$ ). This is more complicated in the case of systems described by irrational transfer function.

Following the line of [9], it is possible to prove that the Bode sensitivity integral theorem can be extended to our case and gives the following constraint:

$$
\int_{0}^{\infty} \log |S(j \omega)| d \omega=0
$$

if the closed loop system is internally stable.

Rather surprisingly, as in the case of rational systems with a time-delay [10], the Bode integral does not imposes supplementary constraint on the sensitivity integral other than the one already obtained for stable minimum phase systems. As proved by Horowitz [11], the real constraint concerns the stability of $S$. Actually, he proved that the frequency range where the open-loop system $L(s)=G(s) K(s)$ has a gain greater than one is limited by a given frequency $\omega_{s}[11$, chap. 7.12.]. In our case, considering that the system is similar to a time-delay system, an approximate value of this frequency is given by $\omega_{c}$ such that $\arg G\left(j \omega_{c}\right)=-\pi$. Since

$$
\arg G(j \omega)=-\frac{X}{\sqrt{2 D}} \sqrt{-\frac{C^{2}}{4 D}+\sqrt{\frac{C^{4}}{16 D^{2}}+\omega^{2}}}
$$

then,

$$
\omega_{s} \approx \frac{\pi C}{X} \sqrt{1+\frac{4 \pi^{2} D^{2}}{C^{2} X^{2}}}=1.9210^{-4} \mathrm{rad} / \mathrm{s}
$$

This value is obtained by considering that the Hayami is fully an inner factor, which is not the case. Therefore, as some phase is given by the outer part, the real upper bound is higher than this value.

\subsection{Pure sensitivity problem}

Another way to examine this problem is to restate the considered problem as an optimization problem. As proved by Zames [16], the sensitivity problem can be recast as a minimization of a weighted $H_{\infty}$ norm problem. In our case, we define a weighting function $W_{1}$ which allows to specify the frequency range where attenuation can be achieved, solve the following problem:

$$
\mu=\inf _{K}\left\|W_{1} S\right\|_{\infty}
$$

While $\mu<1$, we reshape $W_{1}$ in order to increase the bandwidth where $W_{1}$ is greater than one, until $\mu$ is close to one.

The pure sensitivity problem (5) is hard to solve in an approximative way (see [14, sec. 4]), but in our case, linked to the existence of structural bandwidth constraints, it is possible to use a well-posed mixed sensitivity problem to approximate the problem. For the sake of brevity, we do not expose the procedure for this particular problem, since the same one is used in the sequel, with a more realistic constraint. The figure 4 is obtained with the procedure proposed by Rodriguez in [14]. The optimal controller is achieved with an accuracy of $\epsilon=0.1$. The maximum performance obtained is $\omega_{s}=2.410^{-4} \mathrm{rad} / \mathrm{s}$, which is compatible with the value (4) given above.

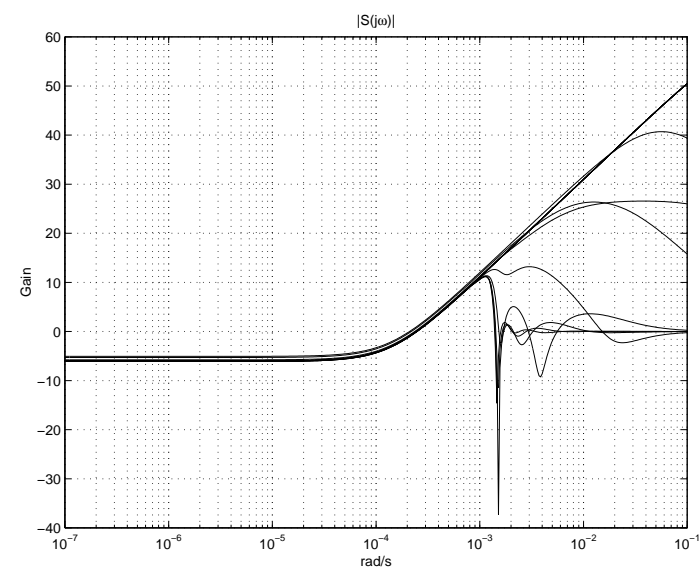

Figure 4: Sensitivity functions for different rational approximations and weight $1 /\left|W_{1}(j \omega)\right|$

\subsection{A constructive approach}

In the previous section, we obtained a hard bound without any classical constraint, like gain margin, phase margin and delay margins. In this section, we now consider a more realistic $H_{\infty}$ criteria, which explicitly shapes the controller under direct constraints on the control. As the original system $G(s)$ is described by an irrational transfer function, we compute a rational approximation $G_{n}(s)$ which can be used to solve the control problem stated above. 
The problem of finding the best achievable performance for open-channel hydraulic systems is considered in the $H_{\infty}$ framework: we will solve a mixed sensitivity problem with constraints on $S$ and $K S$.

The controller $K$ has to reject non measured perturbations (here the withdrawals $w$ ). This can be stated as an mixed sensitivity optimal control problem, using the $H_{\infty}$ norm:

$$
\mu=\inf _{K}\left\|\left(\begin{array}{c}
W_{1} S \\
W_{2} K S
\end{array}\right)\right\|_{\infty}
$$

where $W_{1}$ and $W_{2}$ are weighting functions taking into account performance and robustness requirements. We shape $W_{1}$ and $W_{2}$ in order to obtain at least a gain margin of $9 \mathrm{~dB}$, a phase margin of 65 degrees and a delay margin of $6 \mathrm{~h}$ when $\mu \leq 1$.

When the plant is described by a rational transfer function, this problem is fully understood and solved (see e.g. [7]). Solutions exist for irrational transfer functions (see e.g. [13, 15] and references therein). Unfortunately, the required conditions in [15] are not satisfied in our case since no rational weighting function $W_{2}$ exists such that $W_{2} G_{o}$ and $\left(W_{2} G_{o}\right)^{-1}$ belong to $H_{\infty}$, where $G_{O}$ is the outer factor of $G$.

Following the procedure proposed by Rodriguez [14], we can use a rational approximation to obtain an approximate solution with a guaranteed bound.

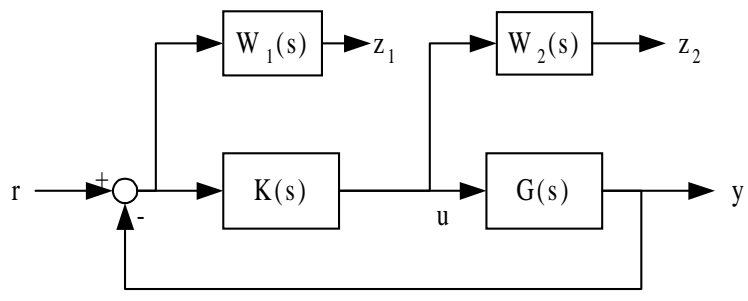

Figure 5: Mixed sensitivity problem

Rational approximation: In the sequel, we will use rational approximation models in order to solve optimization problems. Stable rational approximations of Hayami transfer function are obtained by mean square rational approximations of various orders on a finite frequency range. The error for different orders is plotted on figure 6 .

The $10^{\text {th }}$ order approximation model gives an error of $-90 \mathrm{~dB}$, which corresponds to $310^{-5}$.

Mixed sensitivity controller design: Let us define

$$
B=\frac{1}{\left\|W_{2}\right\|_{\infty}}\left(\left\|W_{1}\right\|_{\infty}+\epsilon\right)
$$

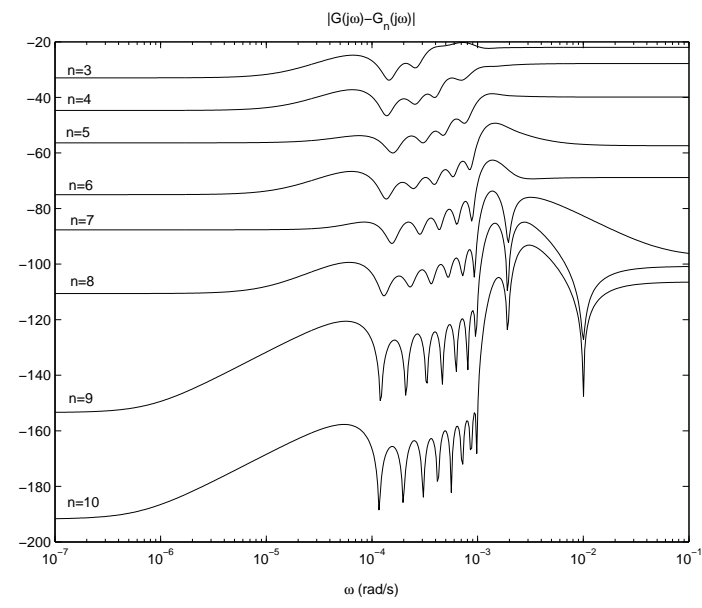

Figure 6: Error $\left|G-G_{n}\right|$ for different orders of approximation $n$

and let us assume

$$
\left\|G_{n}-G\right\|_{\infty} \leq \delta
$$

where $\delta$ is such that :

$$
\delta=\min \left\{\frac{\epsilon}{\left\|W_{1}\right\|_{\infty} B}, \frac{\epsilon}{B}, \frac{1}{B}\right\}
$$

We define below optimal and suboptimal solutions of our problem in the infinite-dimensional case and in the rational case. The optimal solution is defined by:

$$
\mu_{o p t}=\inf _{Q \in H_{\infty}}\left\|\left(\begin{array}{c}
W_{1}(1+G Q) \\
W_{2} Q
\end{array}\right)\right\|_{\infty}
$$

where $Q=K(1+K G)^{-1}$ is the Youla parameter and assumed stable,

and a suboptimal solution $Q_{o}$ such that

$$
\left\|\left(\begin{array}{c}
W_{1}\left(1+G Q_{o}\right) \\
W_{2} Q_{o}
\end{array}\right)\right\|_{\infty} \leq \mu_{o p t}+\epsilon
$$

Moreover, let us define the sub-optimal solution built on the basis of the rational approximation $G_{n}$ of $G$ :

$$
\left\|\left(\begin{array}{c}
W_{1}\left(1+G_{n} Q_{n}\right) \\
W_{2} Q_{n}
\end{array}\right)\right\|_{\infty} \leq \mu_{n}+\epsilon
$$

where $\mu_{n}$ is the optimal value of the $H_{\infty}$ norm:

$$
\mu_{n}=\inf _{Q \in R H_{\infty}}\left\|\left(\begin{array}{c}
W_{1}\left(1+G_{n} Q\right) \\
W_{2} Q
\end{array}\right)\right\|_{\infty}
$$

Following theorem 3.1 in [14], we have the following inequality

$$
\left|\mu_{o p t}-\mu_{n}\right| \leq 2 \epsilon
$$


In our specific case, one has $B=2, \epsilon=310^{-5}$ and then for the $10^{\text {th }}$ order approximation of $G$, we have

$$
\left|\mu_{\text {opt }}-\mu_{n}\right| \leq 610^{-5}
$$

Figure 7 shows the different values of $Q_{n}$ when the order of the approximation increase. Until the $8^{\text {th }}$ order approximation, the solutions remain very close.

The result obtained in this case concerning the maximum performance problem is $\omega_{s}=4.3610^{-5} \mathrm{rad} / \mathrm{s}$. We also get a controller achieving the following robustness margins: a gain margin of $9.13 \mathrm{~dB}$, a phase margin of $67 \mathrm{deg}$ and a delay margin of 7 hours.

$Q_{n}$
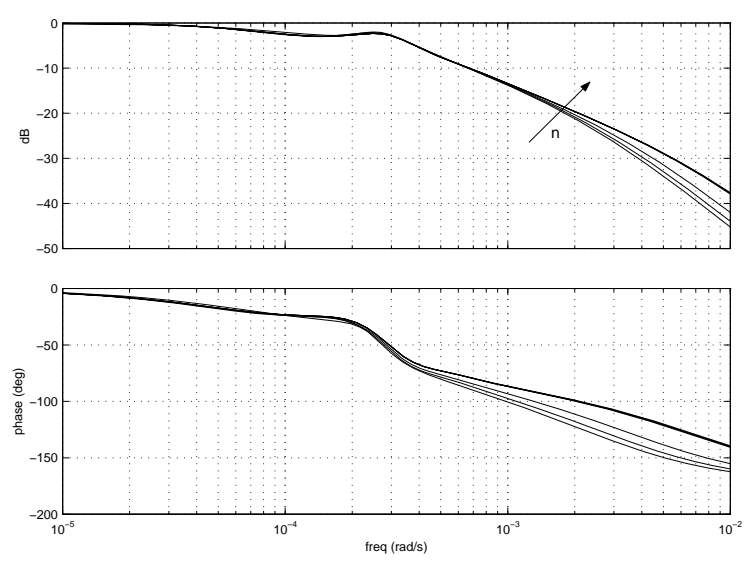

Figure 7: Youla parameter $Q$ for different orders of approximation $n$

\subsection{A qualitative approach using Bode's ideal} transfer function

The results obtained by the numerical approach above can be compared to the ones given by a heuristic approach based on Bode's ideal transfer function. Following the results of Aström [1], we give a priori bounds on the maximum achievable bandwidth using two requirements, one in terms of phase margin, the other one in terms of modulus margin.

Phase margin limitations: The process is factored as $G(s)=G_{m p}(s) G_{n m p}(s)$ where $G_{m p}$ is the minimum phase part and $G_{n m p}$ the non-minimum phase part, with $\left|G_{n m p}(j \omega)\right|=1$.

The open-loop transfer function $L(s)=G_{m p}(s) K(s)$ is supposed to have the ideal shape suggested by Bode $[2]$ :

$$
L(s)=\left(\frac{s}{\omega_{g c}}\right)^{n_{g c}}
$$

$\omega_{g c}$ is the gain cross-over frequency, smallest frequency where $\left|L\left(j \omega_{g c}\right)\right|=1$. The parameter $n_{g c}$ is the slope at the cross-over frequency. The transfer function (6) has the properties that $d \log |L(j \omega)| / d \log \omega=n_{g c}$ and $\arg L(j \omega)=n_{g c} \pi / 2$.

The inequality for the gain cross-over frequency is then obtained as follows. Requiring a phase margin of $\phi_{m}$ leads to

$$
\arg L(j \omega)=\arg G_{n m p}(j \omega)+n_{g c} \frac{\pi}{2} \geq-\pi+\phi_{m}
$$

The cross-over frequency then satisfies the inequality

$$
\arg G_{n m p}\left(j \omega_{g c}\right) \geq-\pi+\phi_{m}-n_{g c} \frac{\pi}{2}
$$

For the Hayami transfer function, the cross-over inequality (7) leads to

$$
\frac{X}{\sqrt{2 D}} \sqrt{-\frac{C^{2}}{4 D}+\sqrt{\frac{C^{4}}{16 D^{2}}+\omega_{g c}^{2}}} \leq \pi+n_{g c} \frac{\pi}{2}-\phi_{m}=2 \alpha_{m}
$$

which is equivalent to

$$
\omega_{g c} \leq \frac{2 \alpha_{m} C}{X} \sqrt{1+\frac{16 \alpha_{m}^{2} D^{2}}{C^{2} X^{2}}}
$$

As $C$ and $D$ depend explicitly on the discharge $Q_{r}$, it is possible to evaluate the limitation (see figure 8 , where $n_{g c}=-1$ and the required phase margin is $\left.\phi_{m}=\pi / 4\right)$. One observes that the maximal bandwidth decreases when the discharge decreases and when the reach length increases. This is conform to physical observations, as the delay increases in these cases.

For a reference discharge $Q_{r}=2 \mathrm{~m}^{3} / \mathrm{s}$, the obtained bound is $\omega_{g c}=4.6510^{-5}$, which is coherent with the value obtained numerically.

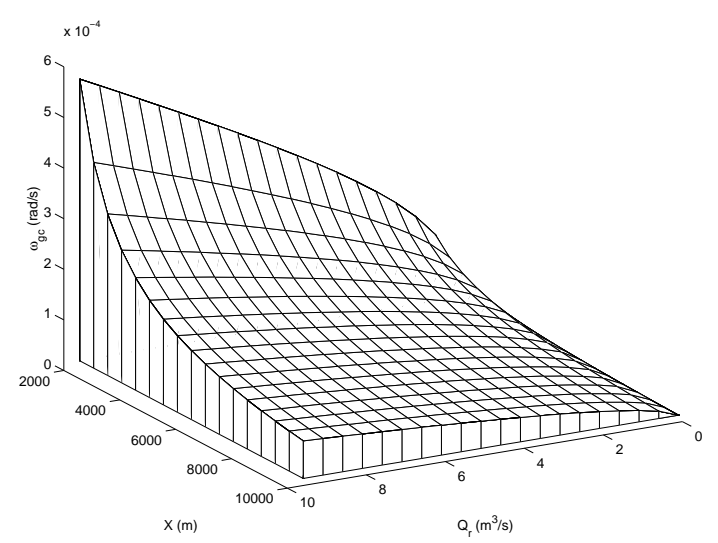

Figure 8: Variation of the maximal bandwidth $\omega_{g c}$ for Hayami transfer function with the discharge $Q_{r}$ and the length of the reach $X$ 
Modulus margin limitations: Following Aström [1], we investigated the relation between the cross-over frequency and the maximal values of the sensitivity function $S=(1+L)^{-1}$ and the complementary sensitivity function $T=L(1+L)^{-1}$, denoted by:

$$
\begin{aligned}
& M_{s}=\max _{\omega}|S(j \omega)| \\
& M_{t}=\max _{\omega}|T(j \omega)|
\end{aligned}
$$

We performed a numerical study to evaluate the limitation on the cross-over frequency $\omega_{g c}$ implied by requirements on $M_{s}$ and $M_{t}$ for Hayami transfer function. The maximum values of $|S(j \omega)|$ and $|T(j \omega)|$ are evaluated for different values of $\omega_{g c}$. The result is given as a function of the "normalized" frequency $\omega_{g c} X / C$ $(X / C$ is a rough estimate of the time-delay of Hayami transfer function).

In order to have $M_{s} \leq 2$ and $M_{t} \leq 2$, the limitation on $\omega_{g c}$ is:

$$
\omega_{g c} \leq 0.7 \frac{C}{X}=4.1310^{-5} \mathrm{rad} / \mathrm{s}
$$

Which is once again coherent with the values obtained previously.

\section{Conclusion}

This paper has shown that the optimal performance of feedback controlled open-channel systems represented by a linear advection-diffusion equation is structurally limited. The maximal achievable bandwidth over which the sensitivity function stays below one is evaluated using different tools. An approximate value is computed with stability considerations. A constructive approach is then performed by designing firstly a pure sensitivity, then a mixed sensitivity $H_{\infty}$ optimal controller on a rational approximation of the system. The mixed sensitivity controller exhibits good robustness margins, which are needed in an application perspective. This numerical approach gives results similar to the ones obtained by a qualitative approach using Bode's ideal loop transfer function. Using the ideal loop transfer function introduced by Bode, we obtained constraint on the maximum achievable bandwidth for open-channel hydraulic systems described by Hayami equation (2) for prescribed phase margin and for modulus margins requirements. In the case of prescribed phase margins, the bound is obtained analytically, which enables to give a priori bounds for a whole family of systems.

This result validates the approximation approach widely used by hydraulic engineers when designing a controller for such systems. It also enables to give a priori bound on the bandwidth over which one should approximate the system, which is important for the rational approximation problem.

\section{Acknowledgement}

This work was supported by the joint research program INRA/Cemagref ASS AQUAE $n^{\circ} 02$ on the control of delayed hydraulic systems.

\section{References}

[1] K.J. Aström. Limitations on control system performance. European Journal of Control, 6:1-19, 2000.

[2] H.W. Bode. Network Analysis and Feedback Amplifier Design. Van Nostrand, New-York, 1945.

[3] F.M. Callier and C.A. Desoer. An algebra of transfer functions for distributed linear time-invariant systems. IEEE Trans. Circuits and Systems, CAS-25(9):651-662, 1978.

[4] V.T. Chow. Open-channel Hydraulics. McGraw-Hill Book Company, New York, 1988. 680 p.

[5] R. Curtain and Y. Zhou. A weighted mixedsensitivity $H_{\infty}$-control design for irrational transfer matrices. IEEE Trans. Aut. Contr., 41(9):1312-1321, 1996.

[6] J.C.I. Dooge, J.J. Napiórkowski, and W.G. Strupczewski. The linear downstream response of a generalized uniform channel. Acta Geophysica Polonica, XXXV(3):279-293, 1987.

[7] J.C. Doyle, K. Glover, P.P. Khargonekar, and B.A. Francis. State-space solutions to standard $H_{2}$ and $H_{\infty}$ control problems. IEEE Trans. Aut. Contr., 34(8):831-847, 1989.

[8] J.S. Freudenberg and D.P. Looze. Right half plane poles and zeros and design tradeoffs in feedback systems. IEEE Trans. Aut. Contr., 30(6):555-565, 1985.

[9] J.S. Freudenberg and D.P. Looze. Inherent feedback limitations for distributed systems. In IFAC 10th World Congress, pages 303-307, 1987.

[10] J.S. Freudenberg and D.P. Looze. A sensitivity tradeoff for plants with time delay. IEEE Trans. Aut. Contr., 32(2):99-104, 1987.

[11] I.M. Horowitz. Synthesis of Feedback Systems. Academic Press, New York, 1963.

[12] X. Litrico and D. Georges. Robust continuous-time and discrete-time flow control of a dam-river system (I): Modelling. Applied Math. Mod., 23(11):809-827, 1999.

[13] H. Özbay. $H_{\infty}$ optimal controller design for a class of distributed parameter systems. Int. Journal of Control, 58(4):739-782, 1993.

[14] A.A. Rodriguez. $\mathcal{H}^{\infty}$ sensitivity and mixedsensitivity optimization for stable multivariable infinitedimensional systems. In Proc. of the 35th IEEE Conf. on Decision and Control, p. 4169 - 4174, 1995.

[15] O. Toker and H. Özbay. $H_{\infty}$ Optimal and Suboptimal Controllers for Infinite Dimensional SISO Plants. IEEE Trans. Aut. Contr., 40(4):751-755, 1995.

[16] G. Zames. Feedback and optimal sensitivity: Model reference transformations, multiplicative seminorms, and approximate inverses. IEEE Trans. Aut. Contr., 26(2):301$320,1981$. 